\title{
THE INTERACTION BETWEEN SHORT OCEAN SWELL AND TRANSIENT LONG WAVES: DISSIPATIVE AND NONLINEAR EFFECTS
}

\author{
James M. Kaihatu ${ }^{1}$, Deirdre Devery ${ }^{2}$, Richard J. Erwin ${ }^{3}$, John T. Goertz ${ }^{4}$
}

\begin{abstract}
The dissipation and nonlinear effects of random swell interaction with transient long waves are studied. Results from a laboratory experiment in which random swell was generated both with and without a co-existing transient long wave are analyzed. An instantaneous dissipation mechanism for estimating both instantaneous and bulk dissipation from data is used to determine the characteristics of dissipation for both cases. Fourier analysis of the free surface measurements and dissipation estimates reveals that the presence of the transient long wave does not have an appreciable impact on the known dissipation characteristics of random swell. However, the use of wavelet analysis, centered on the long wave in the time series, shows that the dissipation characteristics of the combined short-long wave signals deviate considerably from that of swell alone, indicating that smearing of the long wave signal by the Fourier analysis is sufficiently strong to affect dissipation estimates. A wavelet-based bispectral algorithm is used to determine the nonlinear wave-wave coupling in both swell and combined swell-long wave signals; the results indicate that there can be broader ranges of frequencies in which nonlinear coupling is present for the case of the combined short-long wave signal.
\end{abstract}

Keywords: long waves, tsunamis, wavelet analysis, dissipation, bispectra

\section{INTRODUCTION}

Tsunamis are long wave disturbances in the ocean which are caused by seafloor movement. These can be due to seismic movement of the seafloor, subsurface landslides, or similar. The destructive power of tsunamis has been well documented, particularly after the 2004 Indian Ocean and 2011 Japanese tsunamis. Study of tsunamis, particularly in the laboratory, has often been done in isolation from other oceanographic effects (swell band waves, sediment, tides, etc.) and thus their interaction with other elements of the general oceanographic environment is little studied. This has changed recently; for example, Kowalik et al. (2006) and Constantin and Johnson (2008) have investigated the added effect that tides and background vorticity, respectively, have on the tsunami.

Given this change in focus, we consider the possibility that random surface swell can interact with the underlying transient long wave (a proxy for a tsunami) in a non-negligible fashion. This was considered earlier by Kaihatu and El Safty (2011), who investigated the effect of surface swell on the breaking location of the long wave. In this study, we carry this analysis further by performing detailed dissipation calculations on the laboratory data to investigate whether the presence of the transient long waves disrupts the dissipation and nonlinear characteristics known to be present in random swell.

\section{LABORATORY EXPERIMENT}

The experiments were sited at the Tsunami Wave Basin at Oregon State University, Corvallis, Oregon, USA during March 2010. The basin measures $48.8 \mathrm{~m}$ long, $26.5 \mathrm{~m}$ wide and $2.1 \mathrm{~m}$ deep. The maximum water depth for the experiment was $0.75 \mathrm{~m}$. The bathymetry consisted of a flat bottom $(0 \leq x \leq 10 \mathrm{~m}$ where $x=0$ at the wavemaker and positive toward the shoreline), merging with a slope of $1: 15$ $(10 m \leq x \leq 17.5 m)$, and then a slope of $1: 30(17.5 m \leq x \leq 25 m)$. A piston wave maker consisting of 29 individual paddles was used to generate waves. Free surface elevation data were recorded using wire resistance gages, while near bottom velocities were measured with acoustic Doppler velocimeters (ADVs); both were mounted to a movable bridge which was relocated to various measurement stations down the length of the tank. Data were taken at 22 locations, ranging from $x=7.35 m$ to $x=23.18 m$ (in increments of $0.72 \mathrm{~m}$ over the slope and $1.44 \mathrm{~m}$ over the flat bottom) away from the wavemaker. Overhead video imagery from two web cameras were also recorded.The most nearshore gage was located in a water depth of $0.061 \mathrm{~m}$.

The tests consisted of random waves of varying heights and periods, interspersed with a transient long wave. Table 1 shows the test conditions. The tests were conducted in the following configurations:

1. Transient long wave only

\footnotetext{
${ }^{1}$ Associate Professor, Zachry Department of Civil Engineering, Texas A\&M University, College Station, TX, USA

${ }^{2}$ Graduate Research Engineer, Zachry Department of Civil Engineering, Texas A\&M University, College Station, TX, USA

${ }^{3}$ Undergraduate Research Assistant, Zachry Department of Civil Engineering, Texas A\&M University, College Station, TX, USA

${ }^{4}$ Graduate Research Assistant, Zachry Department of Civil Engineering, Texas A\&M University, College Station, TX, USA
} 
2. Each swell condition only

3. Each swell condition with the long wave inserted in the middle of the wave paddle time series

4. Each swell condition with the long wave at the end of the time series.

The long wave (in the form of a solitary wave) had a nominal height of $0.3 \mathrm{~m}$. The actual height of the long wave depended on the significant waveheight of the random swell; since the entire stroke length of the piston wavemaker was used, higher swell corresponded to lower long waves. To build up statistics on the swell, both swell and swell-long wave experiments were run for approximately five minutes. Some representative time series for conditions with and without an included long wave is shown in Figure 1.

\section{ANALYSIS}

Detailed analyses to date have been limited to the free surface elevation data, though initial analyses of the ADV data have begun. Previous analyses of the data by Kaihatu and El Safty (2011) focused on the location of breaking of the long wave; it was seen that the long wave broke further offshore (in deeper water) with a concurrent short wave swell than if no swell were present. Wavelet transforms (e.g., Chui 1992) were used to obtain the time-frequency spectra; strong energy transfer to high frequencies was seen in the spectra contemporaneous with the passage of the long wave through the time series. The HilbertHuang transform (Huang et al. 1998) was then used to separate out the motions into various time scales (modes). By combination of adjacent modes, the free surface motion was divided into "long" wave and "short" wave bands. By calculating the steepness of the wavefield in the individual bands, they determined that the maximum steepness of the wavefield appeared to reside in the short wave band, and hypothesized that the presence of swell might serve as a mechanism for making the transfer of energy to high frequencies during breaking more efficient.

In this study, we investigate the breaking characteristics deduced using the methodology of Kaihatu et al. (2007), which links local wave front-face steepness to instantaneous dissipation estimates. We also investigate linkages to nonlinear wave-wave interaction via the wavelet bicoherence (Dong et al. 2008).

\section{Wave Breaking - Fourier Analysis}

Prior studies of random wave breaking have focused on probabilistic descriptions of bulk dissipation (e.g. Battjes and Janssen 1978; Thornton and Guza 1983; Janssen and Battjes 2007), an approach which suits linear phase-averaged models of wave propagation (e.g. Booij et al. 1999). Incorporation of these models into phase-resolved descriptions which include the quadratic nonlinearity endemic to shallow water wave propagation (Agnon et al. 1993; Kaihatu and Kirby 1995) require more care since the frequency distribution of dissipation needs to be specified. It was hypothesized by Kirby and Kaihatu (1996), and further confirmed by Chen et al. (1997) and Kaihatu et al. (2007), that a frequency squared weighting of dissipation over the spectrum is both physically justifiable and accurately captures wave shape statistics.

In the process of confirming this frequency distribution, Kaihatu et al. (2007) used a method for inferring instantaneous dissipation (Zelt 1991) from random wave data which, when processed as time series to obtain spectra, showed that the deduced dissipation rate reflected in the data trends as frequency squared. The instantaneous dissipation, adapted from Zelt (1991) to accommodate temporal measurements of free surface elevation, is:

$$
\epsilon=-\rho\left(\frac{\eta}{h}\right)\left(v_{b} \eta_{t}\right)_{t}
$$

where $\rho$ is the density of water, $\eta$ is the free surface elevation, $h$ is the water depth, and the subscript $t$ refers to differentiation with respect to time. The eddy viscosity $v_{b}$ is defined as:

$$
v_{b}=B \gamma^{2} h \eta_{t}
$$

where:

$$
\begin{aligned}
B & =1 \quad \eta_{t} \geq 2 \eta_{t}^{*} \\
B & =\frac{\eta_{t}}{\eta}-1 \quad \eta_{t}^{*} \leq \eta_{t}<2 \eta_{t}^{*} \\
B & =0 \quad \eta_{t}<\eta_{t}^{*}
\end{aligned}
$$


and

$$
\eta_{t}^{*}=\Gamma \sqrt{g h}
$$

In (4), the parameter $\Gamma=0.3$ corresponded to the case of solitary waves breaking over a flat bottom (Zelt 1991). Goertz et al. (2012, this conference) has shown that a value of $\Gamma=0.9$ best matches estimates of probabilistic dissipation from established models calibrated with measurements of wave propagation over a steep slope. (We note here that Zelt (1991) had apparently not intended that $\Gamma$ be any value other than 0.3 .)

Assuming a linear shoaling relationship balancing the evolution of the complex amplitude of the free surface elevation $A_{n}$ and the dissipation:

$$
A_{n x}+\frac{C_{g n, x}}{2 C_{g n}} A_{n}=-\alpha_{n} A_{n}
$$

where the subscript $n$ refers to the $n^{\text {th }}$ frequency component in the spectrum, $C_{g}$ is the group velocity and $\alpha_{n}$ is the dissipation rate coefficient. Converting this to an equation for the smoothed free surface elevation spectrum $S_{\eta}$, the dissipation spectrum $S_{\epsilon}$ and solving for $\alpha_{n}$ (Kirby and Kaihatu 1996):

$$
\alpha_{n}=\frac{1}{\rho g \sqrt{g h}} \frac{1}{\sqrt{2 \Delta f}} \frac{\sqrt{S_{\epsilon}(f)}}{S_{\eta}(f)}
$$

Using this, Kaihatu et al. (2007) showed that the frequency dependence of $\alpha_{n}$ (required to be specified apriori in a modeling context) approaches an $f^{2}$ weighting in the saturated surfzone, but evolves to that value gradually from the offshore. In contrast, Kaihatu and El Safty (2011), on the other hand, showed that this trend was not evident in wave group data due to the presence of the bound long wave, which tended to disrupt this trend.

Akin to prior studies, we apply this analysis to the free surface elevation of the data. In order to capture both the randomness of the wavefield without oversmoothing the effect of the long wave, the time series of free surface elevations was truncated at 2048 points, with the long wave (if present) centered in the time series. Spectra of the free surface elevations and the dissipation, as well as the dissipation rate $\alpha_{n}$, for Swell Test 1 (with long wave) are shown in Figure 2. It can be seen that the dissipation spectra $S_{\epsilon}$ is lower in spectral density for the combined short-long wave case than for the swell in isolation. In addition, the low frequency bands for all three quantities are amplified with the long wave present relative to those of the swell in isolation. This suggests that the long wave is represented in the spectra, and that its signature has survived the Fourier-based analysis.

In a manner similar to the previous study of Kaihatu et al. (2007) we track the evolution of the frequency dependencies of the spectral tails of both $\alpha_{n}$ and the free surface elevation spectra $S_{\eta}(f)$. Also similar to the previous study, we use the frequency range from the peak frequency to half of the Nyquist frequency $f_{N y q}=12.5 \mathrm{~Hz}$. Inspection of Equation (6) reveals that, if $S_{\epsilon}(f)$ were constant with frequency (endemic for a process consisting of successive spikes), an inverse relationship between the frequency dependencies of $\alpha_{n}$ and $S_{\eta}(f)$ must exist. This can be seen in Figure 3. These show that the trend toward an $f^{2}$ frequency dependence for $\alpha_{n}$, seen by Kaihatu et al. (2007) for random wave data, also appears to be the case here for the case with an included transient long wave - at least when standard Fourier analysis techniques are used.

The overall bulk dissipation can be derived using Equation (5):

$$
\frac{\partial\left(E C_{g}\right)}{\partial x}=-D=-\rho g \sum_{n=1}^{N} C_{g n}\left|A_{n}\right|^{2} \alpha_{n}
$$

This allows for estimation of bulk dissipation by summing over all frequencies in the spectrum. We use Equation (7) to determine how the dissipation of the swell is affected by the presence of the long wave. This is shown in Figure 4; a value of $\Gamma=0.9$ is used. For a majority of the domain, the dissipation of the co-existing short and long wave fields is less than that of the swell field by itself. This can be explained by considering the breaking mechanism (2), in which the dissipation is activated by the forward slope face. It is possible that the presence of the long wave is reducing the overall steepness of the wavefield and causing less dissipation. This is the converse observation to that of Kaihatu and El Safty (2011), who hypothesized that the presence of swell increased the steepness of the long wave. 


\section{Wave Breaking - Wavelet Analysis}

Though relatively straightforward to interpret, it can be argued that the use of a standard Fourier analysis is not advisable in this situation. Despite the truncation of the time series to a relatively small segment of the experiment, the effect of highly transient signals can be smeared over frequencies, and details of the interaction are potentially lost. However, some degree of statistical certainty is still required, so sharp resolution is required.

To address this, we also used the Morlet wavelet transform (e.g. Chui, 1992) on the time series data. The wavelet transform is often used for the analysis of highly transient phenomena. It shares many of the same characteristics as Fourier analysis, except that the basis function is a brief oscillation which begins and ends at zero. The width of the wavelet is analogous to the frequency of a sine wave. This form allows localization of the function near the transient part of the signal. To add statistical smoothing, the spectra were averaged over the temporal "width" of the long wave.

We performed the same breaking and dissipation analysis on these wavelet spectra as was done using the Fourier spectra. The disspation rate $\alpha_{n}$ and the free surface elevation spectra $S_{\eta}(f)$ were calculated by the expressions above, and the slopes of the spectra calculated using the same frequency ranges as before. The result for Swell 1 without the long wave is in Figure 5, while Swell 1 with the long wave is shown in Figure 6. It is evident that, unlike the Fourier-based analysis, the wavelet analysis yields behavior that is quite different between the cases with and without the long wave. While the swell-only cases show a gradual reduction in frequency slopes toward $f^{1}$, the swell-long wave cases display a relatively high degree of evolution, with no clear trend toward an asymptote in the nearshore. The differences between these results and those from the Fourier analysis indicates that the transience of the signal of the long wave has a deep effect on the dissipation characteristics.

\section{Wavelet Bispectra}

The bispectrum is a means for determining the relative strength of nonlinear energy exchange between frequencies in a spectrum. The evolutionary characteristics of bispectra with respect to ocean waves has been long known (e.g. Elgar and Guza 1985). For the present case, in which we have a strong transient signal propagating through a transient signal, we use a bispectral analysis which is able to focus on the transient wave. This uses an algorithm from Dong et al. (2008), who developed a means of calculating bispectra using wavelet analysis rather than Fourier analysis.

The following form for a wavelet transform is assumed:

$$
W(\tau, a)=\int_{-\infty}^{\infty} \eta(t) \Psi_{a, \tau}^{*} d t
$$

where $\tau$ is the time translation, $a$ is the dilation scale and $\Psi_{a, \tau}(t)$ are the wavelets, which serve as the bases over which the time series is expanded. The asterisk denotes complex conjugate. Dong et al. (2008), following Van Milligen et al. (1995), then define the wavelet bispectrum:

$$
B_{x x y}\left(a_{1}, a_{2}\right)=\int_{T} W_{x}\left(\tau, a_{1}\right) W_{x}\left(\tau, a_{2}\right) W_{y}^{*}(\tau, a) d \tau
$$

where the subscripts $x$ and $y$ denote time series incorporated in the wavelet functions. Just as with Fourierbased bispectra (e.g. Elgar and Guza 1985), the frequency-like dilation $a$ follow a summation rule:

$$
\frac{1}{a}=\frac{1}{a_{1}}+\frac{1}{a_{2}}
$$

Correlations between changes in energy level between the three frequency bands comprising the triad are tracked by the bicoherence, the amplitude of the bispectrum. Thus, high levels of bicoherence indicate strong coupling between the triad components.

This analysis technique was applied to the data from the experiment. To avoid smearing the long wave among many frequencies, we limit the analysis in time to the period bracketing the tsunami. One example of this analysis appears in Figures 7 and 8. In each figure, color plots of bicoherence for data taken at a water depth of $0.1 \mathrm{~m}$ are shown - one for the swell in isolation (Figure 7), and one for the combined shortlong wave motion (Figure 8). For the triad of $f=(0.5,1.0,1.5) \mathrm{Hz}$, the combined short-long wave motion shows an enhanced bicoherence relative to that of the swell motion by itself. Th combined short-long wave 
motion also shows much less smearing of bicoherence levels at higher frequencies than that of the swell in isolation, which seems to have bicoherence levels spread over a wide range of frequencies. However, more study of these results is required before any definitive conclusions can be drawn.

\section{CONCLUSION}

The interaction between random swell and a transient long wave is studied, with explicit focus on the dissipation and nonlinear characteristics. Extending the work of Kaihatu and El Safty (2011), we use an eddy viscosity breaking model (Zelt 1991) to deduce the instantaneous dissipation, which could then be used to calculate the bulk dissipation. Using Fourier analysis on the free surface information and the dissipation estimates, we determine that the spectral dissipation characteristics for the combined shortlong wave signals are the same as that for the swell alone; the frequency dependence of the dissipation approaches $f^{2}$ in the nearshore, which Kaihatu et al. (2007) shows as being an appropriate nearshore asymptote in the saturated surf zone. Using wavelet transforms, however, it is apparent that the dissipation characteristics for the combined short-long wave signals are considerably different than for the swell in isolation, indicating that the Fourier analysis may be smearing the influence of the transient long wave over a wide range of frequencies. Finally, a wavelet-based bispectra algorithm (Dong et al. 2008) was used to determine the level of nonlinear coupling between the frequencies in an interacting triad. While some interesting differences were seen between the bicoherence levels of short-long wave motions and those of the short swell alone (including less smearing of bicoherence across frequencies in the case of the combined signal), more study of these results is required before definitive conclusions are drawn.

\section{ACKNOWLEDGEMENTS}

This work was supported by the Civil, Mechanical and Manufacturing Innovation Directorate of the National Science Foundation (Grant No. 0936579). We would like to thank the staff of the Tsunami Wave Basin at the O.H. Hinsdale Laboratory at Oregon State University for their facilitation of the experiments. The help of the Research Experiences for Undergraduates (REU) students - Belynda Alonzo, Kyle Outten and Brianna Schilling - is appreciated. Finally, Dr. Yuxiang Ma of Dalian University of Technology kindly made his wavelet bicoherence code available for our use.

\section{REFERENCES}

Agnon, Y., Sheremet, A., Gonsalves, J., and Stiassnie, M., 1993. Nonlinear evolution of a unidirectional shoaling wave field. Coastal Engineering, 20, 29-58.

Battjes, J.A., and Janssen, J.P.F.M., 1978. Energy loss and set-up due to breaking of random waves. Proceedings of the 14th International Conference on Coastal Engineering, Hamburg, Germany, ASCE, 466-480.

Booij, N., Ris, R.C., and Holthuijsen, L.H., 1999. A third-generation wave model for coastal regions, 1. Model description and validation. Journal of Geophysical Research, 104, 7649-7666.

Chen, Y., Guza, R.T., and Elgar, S., 1997. Modeling spectra of breaking surface waves in shallow water. Journal of Geophysical Research, 102, 25035-25046.

Chui, C., 1992. An Introduction to Wavelets. Academic Press, New York.

Constantin, A., and Johnson, R.S., 2008. Propagation of very long water waves, with vorticity, over variable water depth, with application to tsunamis. Fluid Dynamics Research, 40, 175-211.

Dong, G., Ma, Y., Perlin, M., Ma, X., Yu, B., and Xu, J., 2008. Experimental study of wave-wave nonlinear interactions using the wavelet-based bicoherence. Coastal Engineering, 55, 741-752.

Huang, N.E., Shen, Z., Long, S.R., Wu, M.C., Shih, H.H., Zheng, Q., Yen, N.-C., Tung, C.-C., and Liu, H.H., 1998. The empirical mode decomposition and the Hilbert spectrum for nonlinear and nonstationary time series analysis. Proceedings of the Royal Society of London, Ser. A., 454, 903-995.

Janssen, T.T., and Battjes, J.A., 2007. A note on wave energy dissipation over steep beaches. Coastal Engineering, 54, 711-716.

Kaihatu, J.M., and Kirby, J.T., 1995. Nonlinear transformation of waves in finite water depth. Physics of Fluids, 7, 1903-1914.

Kaihatu, J.M., Veeramony, J., Edwards, K.L., and Kirby, J.T., 2007. Asymptotic behavior of frequency and wavenumber spectra of nearshore shoaling and breaking waves. Journal of Geophysical Research, 112, doi: 10.1020/2006JC003817. 
Kaihatu, J.M., and El Safty, H., 2011. The interaction of tsunamis with ocean swell: an experimental study. Proceedings of the 30th International Conference on Ocean, Offshore and Arctic Engineering OMAE2011, Rotterdam, the Netherlands.

Kirby, J.T., and Kaihatu, J.M., 1996. Structure of frequency domain models for random wave breaking. Proceedings of the 25th International Conference on Coastal Engineering, Orlando, FL, ASCE, 1144-1155.

Kowalik, Z., Proshutinsky, T., and Proshutinsky, A., 2006. Tide-tsunami interactions. Science of Tsunami Hazards, 242-256.

Thornton, E.B., and Guza, R.T., 1983. Transformation of wave height distribution. Journal of Geophysical Research, 88, 5925-5938.

Van Milligen, B.P., Sanchez, E., Estrada, T., Hidalgo, C., Branas, B., Carreras, B., Garcia, L., 1995. Wavelet bicoherence: a new turbulence analysis tool. Physics of Plasmas, 2, 3017-3032.

Zelt, J.A., 1991. The run-up of nonbreaking and breaking solitary waves. Coastal Engineering, 15, 205-246. 


\begin{tabular}{|c|c|c|c|c|c|}
\hline Test & $H_{s}(m)$ & $T_{p}(s)$ & $\mu=k h$ & $\delta=a / h$ & $U_{r}=\delta / \mu^{2}$ \\
\hline 1 & 0.05 & 2 & 1 & 0.033 & 0.033 \\
\hline 2 & 0.05 & 4 & 0.45 & 0.033 & 0.163 \\
\hline 3 & 0.1 & 2 & 1 & 0.067 & 0.067 \\
\hline 4 & 0.1 & 4 & 0.45 & 0.067 & 0.331 \\
\hline
\end{tabular}

Table 1: Swell conditions for laboratory experiment
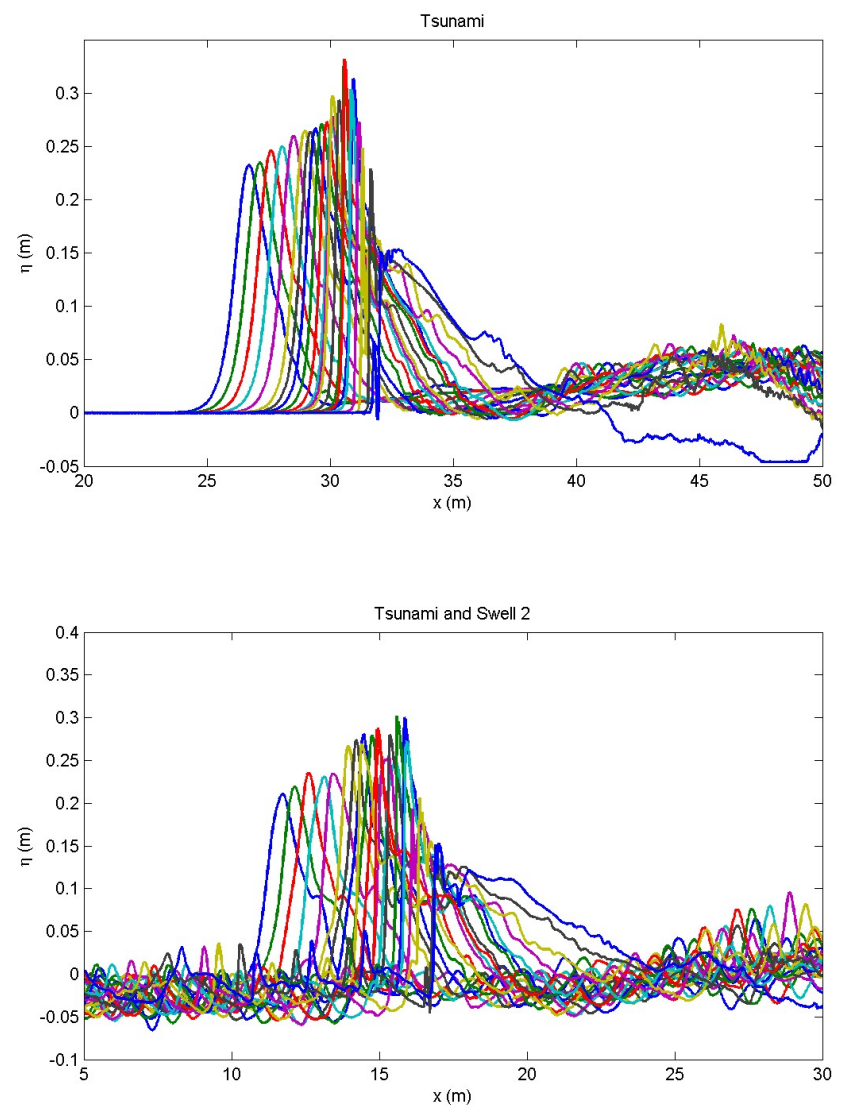

Figure 1: Examples of time series from all gages. Top: Long wave. Bottom: Long wave with random swell 

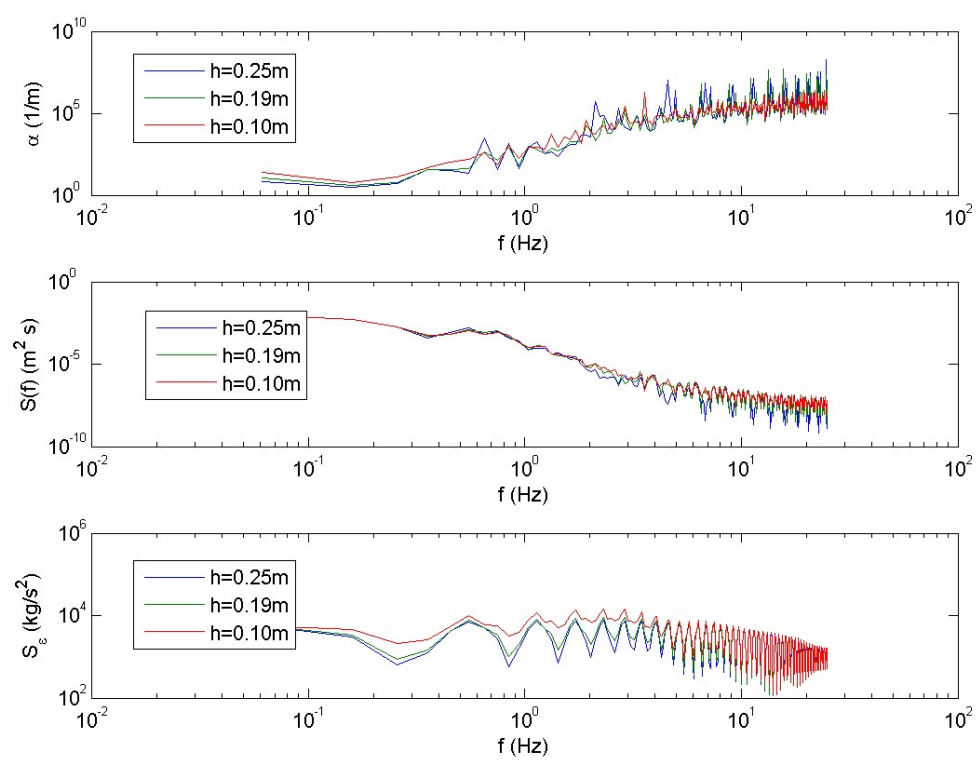

Figure 2: Free surface spectra and dissipation from Test 1 with long wave. Top: $\alpha_{n}$. Middle: Spectra of free surface elevation. Bottom: Spectrum of time series of estimated dissipation $\epsilon$
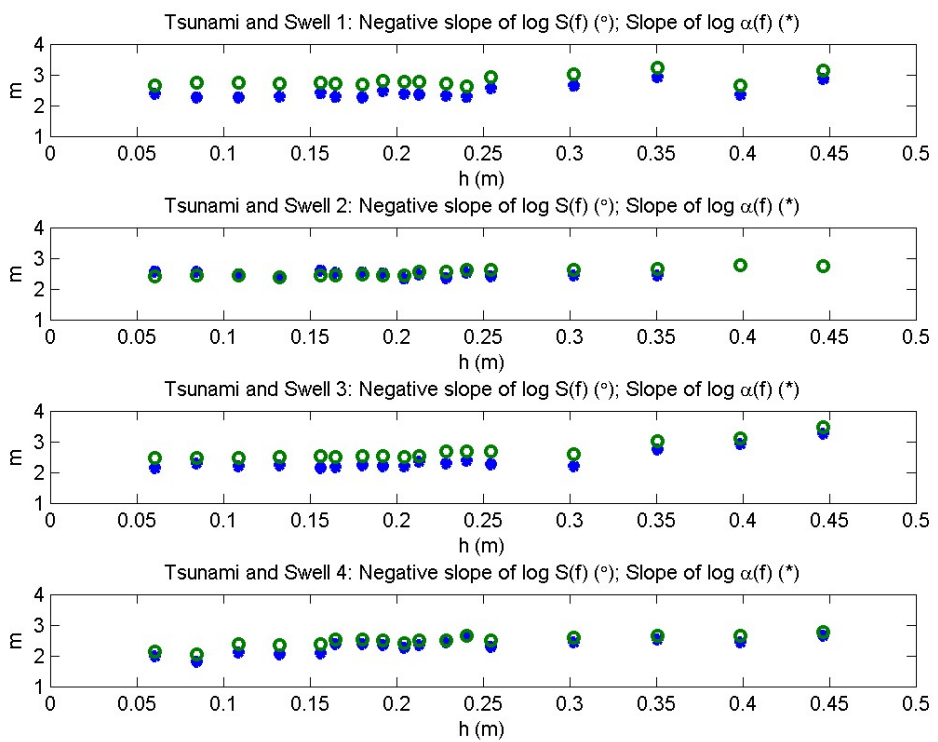

Figure 3: Slope of the spectral tail of $S(f)$ (circles) and $\alpha_{n}$ (asterisks) as a function of water depth. All tests with long wave included. 

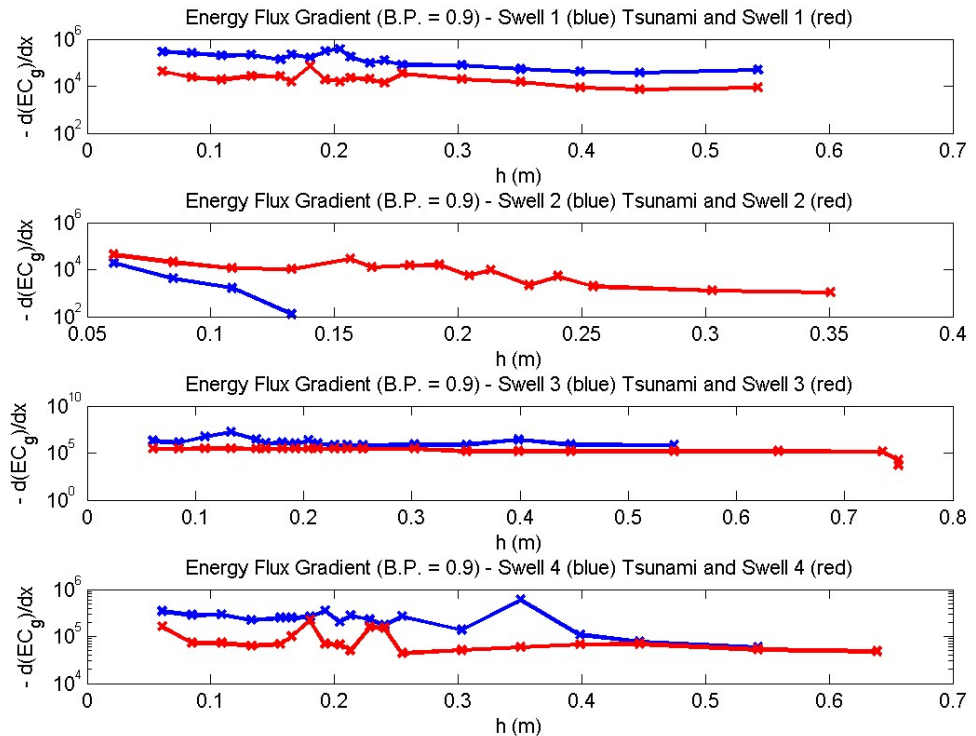

Figure 4: Energy flux gradient for swell only (blue) and swell with long wave (red) $-\Gamma=0.9$ throughout.

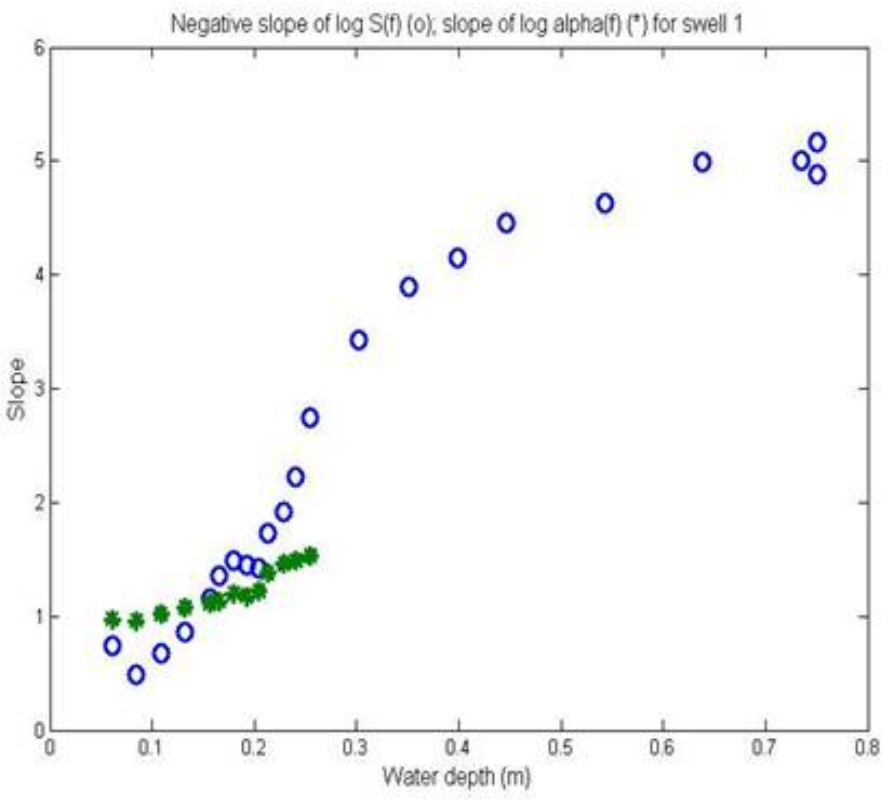

Figure 5: Slope of the spectral tail of $S(f)$ (circles) and $\alpha_{n}$ (asterisks) as a function of water depth: result from wavelet analysis. Swell 1 shown. 


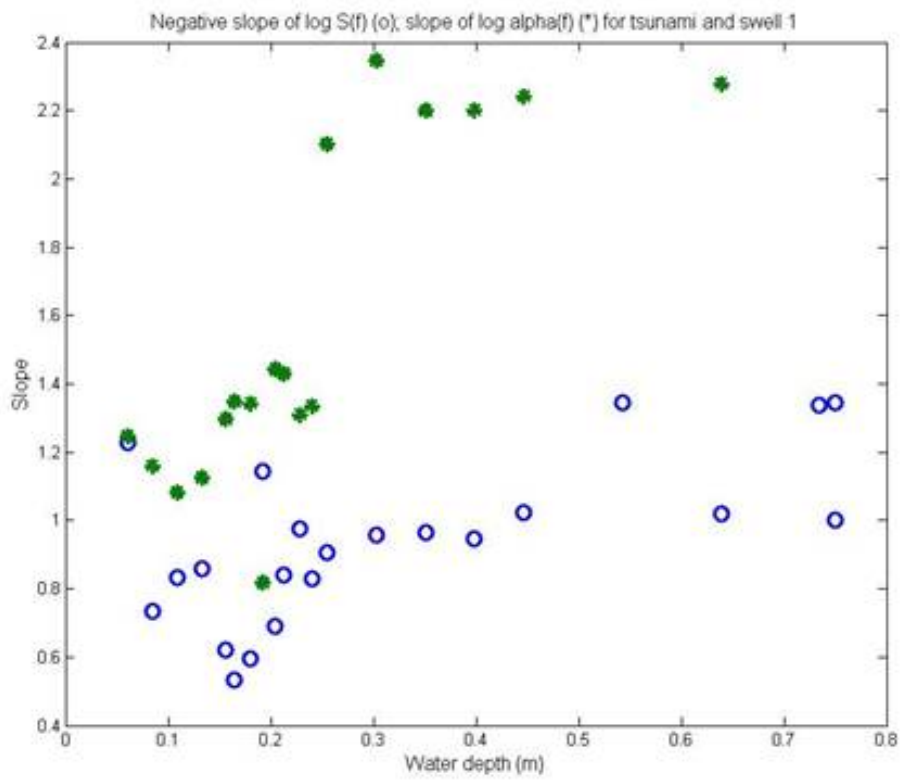

Figure 6: Slope of the spectral tail of $S(f)$ (circles) and $\alpha_{n}$ (asterisks) as a function of water depth: result from wavelet analysis. Swell 1 with long wave shown.

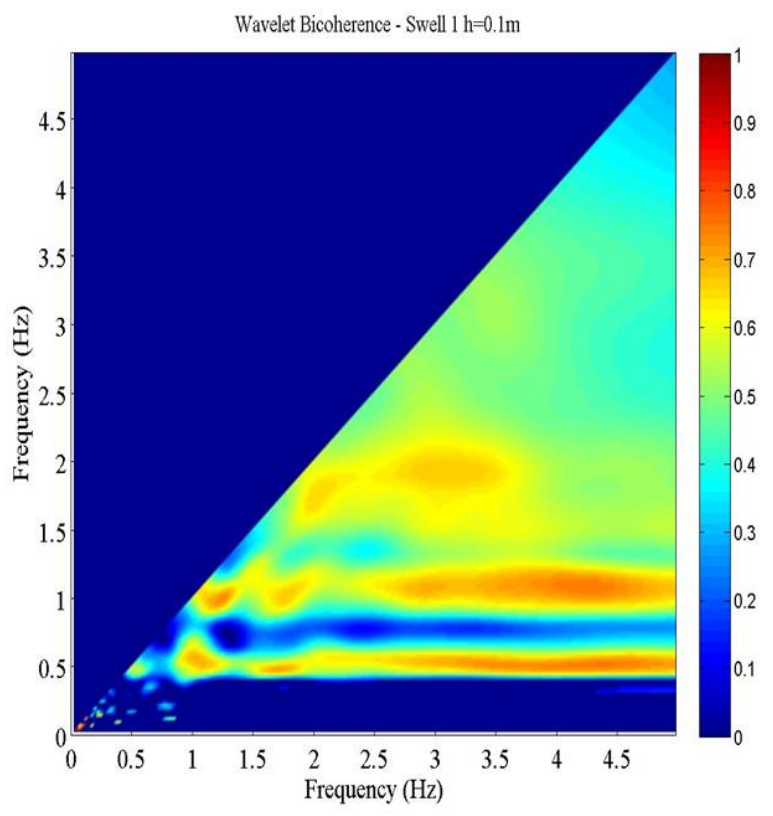

Figure 7: Wavelet bicoherence of free surface elevation data at $h=0.1 \mathrm{~m}$. Swell 1 shown. 


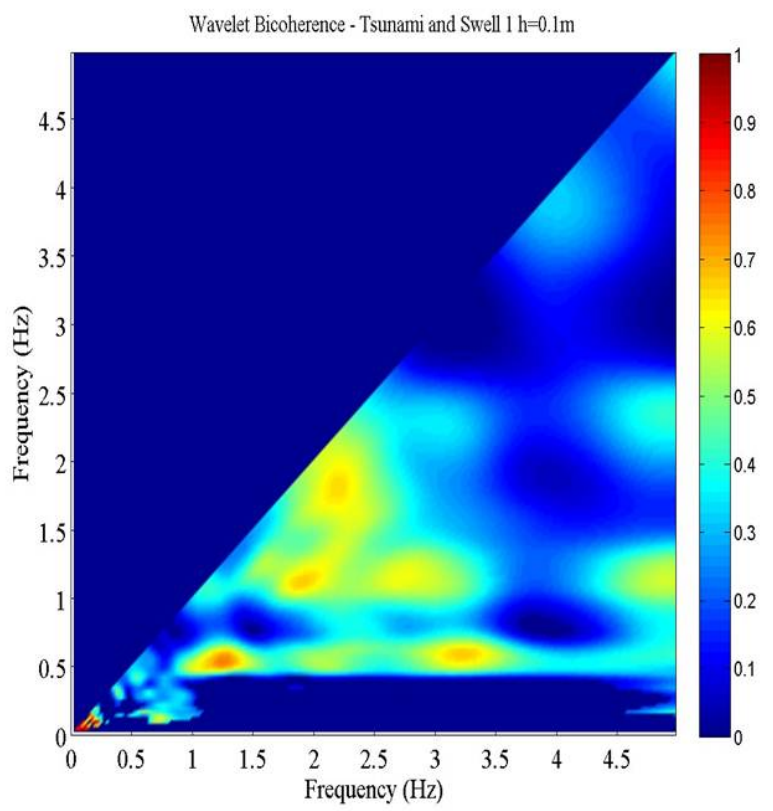

Figure 8: Wavelet bicoherence of free surface elevation data at $h=0.1 \mathrm{~m}$. Swell 1 with long wave shown. 